\title{
VALORACIÓN DE COMUNIDADES DE APRENDIZAJE POR EL VOLUNTARIADO UNIVERSITARIO
}

\section{María Teresa Gómez del Castillo Segurado y Antonio Aguilera Jiménez}

\section{RESUMEN}

En la Universidad de Sevilla venimos colaborando con los centros constituidos como Comunidades de Aprendizaje desde el año 2006, en el que se crearon las primeras. Uno de los modos en que se concreta esta colaboración es facilitando que estudiantes universitarios de cualquier titulación puedan participar como voluntarios en las distintas actuaciones educativas que estos centros llevan a cabo como el trabajo en grupos interactivos, biblioteca tutorizada o tertulias dialógicas, entre otras. Un elemento esencial para la mejora de estas Prácticas de Intervención Psico-educativa en Comunidades de Aprendizaje es la revisión de las actuaciones desarrolladas. Con esa finalidad hemos elaborado dos cuestionarios que los estudiantes deben responder on-line al finalizar cada curso académico: uno sobre la satisfacción del voluntariado y otro sobre la valoración que los estudiantes hacen de las actuaciones educativas de las $\mathrm{CdA}$. En este artículo presentamos ambos instrumentos de manera íntegra así como los resultados obtenidos con ellos tras el curso 2013-14.

Palabras clave: Voluntariado, Comunidades de Aprendizaje, evaluación, satisfacción, prácticas psicoeducativas.

\section{TITLE: ASSESSMENT OF LEARNING COMMUNITIES BY UNIVERSITY VOLUNTEERS}

\section{ABSTRACT}

At the University of Seville we collaborate with schools which have been constituted as Learning Communities since 2006, year in which the first were established. One of the ways in which this collaboration is achieved is by making it easier for students of any degree to participate as volunteers in the various educational activities that these centers carry out such as working in interactive groups, tutoring library or talk gatherings, among others. An essential element for improving these Psycho-Educational Intervention practices in Learning Communities is the review of the actions undertaken. To this end we have developed two questionnaires that students must respond on-line at the end of each academic year: one on volunteers' satisfaction and another on the assessment that students make of the educational actions of Learning Communities. In this paper we present both instruments entirely and the results obtained with them after the 20I3-14 year.

Keywords: Volunteering, Learning Communities, Evaluation, Satisfaction, Psycho-educational Practices.

Correspondencia con los autores: María Teresa Gómez del Castillo Segurado<mgomezdelcastillo@us.es>.Dpto.de Didáctica y Organización Escolar, Univ. de Sevilla. Antonio Aguilera Jiménez < aguijim@us.es>.Dpto. de Psicología Evolutiva y de la Educación, Univ. de Sevilla. Original recibido: 19-12-14. Original aceptado: 20-03-15. 


\section{Introducción}

Una Comunidad de Aprendizaje (en adelante CdA) es un modelo de organización para cualquier centro educativo que quiera dar respuesta a la mejora del rendimiento académico de sus alumnos y prevenir y mejorar los problemas de convivencia a partir de un modelo comunicativo dialógico de aprendizaje. Su fundamentación teórica está cimentada en autores como Freire, Bruner, Habermas o Vygotsky, entre otros (Rodríguez de Guzmán, 20I2).

Los grupos interactivos, las tertulias dialógicas, el diálogo igualitario y la participación de las familias y la comunidad en el funcionamiento de las escuelas, constituyen los ejes fundamentales en torno a los que giran las actuaciones educativas de éxito que se aplican (INCLUD-ED, 20II). Un aspecto esencial de estas actuaciones de éxito es que son universales y transferibles, es decir, que obtienen buenos resultados en todos los contextos (Valls y Padros, 20 I I). Es una propuesta que se aleja de las prácticas de la educación compensatoria, porque no se fundamenta en el "déficit" a compensar de los alumnos, sino en la mejora de la calidad de la enseñanza que se les proporciona (Elboj, Puigdellívol, Soler, y Valls, 2002).

Las primeras CdA se implantaron en Sevilla en 2006, y desde ese curso la Universidad ha colaborado con ellas a través de diversas tareas, una ha sido aportando estudiantes universitarios voluntarios para colaborar con los centros, fundamentalmente en tres tipos de actuaciones de éxito:

- Los grupos interactivos: Agrupaciones heterogéneas de alumnos, constituidas por cuatro o cinco alumnos, de manera que trabajen colaborativamente las actividades propuestas por el profesorado. Para ello cuentan con un voluntario en cada grupo que se encarga de dinamizar y potenciar las interacciones entre los participantes (Gómez del Castillo, Aguilera y Prados, 2015). Cada equipo trabaja en una actividad diferente con adultos distintos durante un periodo de 15 ó 20 minutos. Tras pasar ese tiempo rotan a otra actividad. El grupo debe resolver la actividad a través del diálogo igualitario, tanto si la actividad es grupal como si es individual. Esto quiere decir que son ellos mismos los que se ayudan y resuelven las dudas (Rubia, Jorri y Anguita, 2009). Las tareas propuestas para trabajar en los grupos interactivos son las propias del currículo, trabajando contenidos de cualquier área. 
- Bibliotecas tutorizadas: Se trata de que las bibliotecas se aprovechen como lugares para interaccionar con compañeros, adultos, voluntarios, profesores de manera que se conviertan en espacios de máximo aprovechamiento educativo, donde acudan los alumnos a estudiar, comentar dudas, leer, charlar sobre algún tema (Red Utopía y Educación, 20I2). En la biblioteca tutorizada, por ejemplo, "acuden alumnos y alumnas voluntariamente para poder realizar las tareas escolares, resolver dudas, llenar lagunas académicas" (Aretxaga y Landaluce, 2005, p. 2I4), y pueden estar a cargo del profesorado o de algún voluntario o familiar.

- Tertulias dialógicas: Se trata de la construcción colectiva de significado y conocimiento en base al diálogo igualitario con todos los participantes en la tertulia. Tienen como base las reflexiones, los debates y los argumentos, y también las experiencias de vida cotidiana de los participantes. Consiste en participar en un seminario de formación en el que todos los asistentes (docentes, familiares, voluntarios, etc.) se reúnen para comentar la lectura de un libro en común que se selecciona a principio de curso. Cada sesión se dedica a una parte del libro que todos preparan previamente fuera de la tertulia. Durante la sesión una persona previamente acordada introduce el capítulo leído y a partir de ahí se entabla un diálogo entre todos los asistentes. Normalmente, el libro suele estar relacionado con temas educativos o con obras maestras de la literatura universal. En algunos casos, los menos, pueden ser audiciones y entonces serán tertulias musicales (Aguilera et al, 20I4).

Los centros educativos a los que asisten estos voluntarios son centros escolares reconocidos por la Consejería de Educación de la Junta de Andalucía como Comunidades de Aprendizaje, siendo una de las características definitorias de estas la mayor implicación de profesorado, familiares, alumnado y otros miembros del entorno social en un proyecto educativo compartido donde, como decía Freire (2005, p. 6I) "nadie lo sabe todo, nadie lo ignora todo. Todos sabemos algo, todos ignoramos algo".

En este trabajo mostramos la evaluación que los alumnos universitarios voluntarios en estos centros realizaron al finalizar sus prácticas de la actividad de libre elección "Prácticas de Intervención Psicoeducativa en Comunidades de Aprendizaje" que ha sido ofrecida en el curso 2013-14 de manera interdepartamental, con la participación de profesores de los Departamentos de Psicología Evolutiva y de la Educación y de 
Didáctica y Organización Educativa, de la Universidad de Sevilla, lo que creemos que supone un valor añadido a la calidad universitaria en línea a la mejora e innovación de las prácticas docentes (Imbernón, 20I2). Se propuso para todos los alumnos de la Universidad como actividad de libre configuración, de reconocimiento de créditos o de voluntariado para los que no optasen por ninguna de las dos posibilidades primeras. La gran mayoría de los alumnos fueron estudiantes de la Facultad de Ciencias de la Educación.

La actividad de reconocimiento de créditos (anteriormente asignatura de libre configuración) implica a los estudiantes en actuaciones educativas de éxito y de superación del fracaso escolar. Hemos comprobado todos estos años que la participación en Grupos Interactivos, Tertulias Dialógicas o en otras actividades llevadas a cabo por los estudiantes en las CdA resultan gratificantes personalmente para ellos y muy productivas desde un punto de vista académico. Al inscribirse en la actividad los alumnos se comprometen a realizar las siguientes tareas (Aguilera, Ignacio, Prados, Sánchez, Gómez del Castillo, Ordóñez y Rodríguez, 20I4):

I) Asistencia a un seminario de 30 horas aproximadamente en modalidad presencial o semipresencial donde se exponen los fundamentos teóricos de las CdA.

2) Desplazarse a una CdA durante 3 horas semanales para colaborar como voluntario en las actuaciones educativas de éxito que se lleven a cabo en el centro elegido.

3) Asistir a una Tertulia Dialógica (pedagógica o literaria) de 2 horas al mes que se desarrollará en el mismo centro en el que se desarrolla la actividad del estudiante.

4) Contestar, al finalizar la colaboración en CdA, a dos cuestionarios de revisión y evaluación diseñados a tal fin.

Es este último punto el que centrará el trabajo que presentamos aquí. Los resultados de estos dos cuestionarios destinados al voluntariado universitario: El primero "Cuestionario de SATISFACCIÓN del voluntariado universitario en CdA" (SAVU-CdA) (Aguilera, Gómez del Castillo y Prados, 20I4a) y el segundo "Cuestionario de VALORACIÓN de CdA por el voluntariado universitario" (VAVU-CdA) ) (Aguilera, Gómez del Castillo y Prados, 20l4b). Fueron elaborados a partir de las memorias comentadas de los propios alumnos de cursos anteriores, y que a partir de un trabajo de categorización y unas pruebas 
de fiabilidad se comenzaron a utilizar en el curso 20 I3-I4 (Aguilera y Gómez del Castillo, 20I4).

Una de las motivaciones que tenemos para impulsar desde la universidad las prácticas en actuaciones de éxito educativo a través de la participación de los estudiantes en $\mathrm{CdA}$, es intentar contribuir a una mayor calidad de nuestra universidad, especialmente de los profesionales que intervendrán en los ámbitos educativos. Para ello, creemos que una buena formación tiene que partir de presentar investigaciones a los estudiantes que reflejen los éxitos y los mecanismos de transformación de la educación que existen y que han demostrado que funcionan a nivel internacional (Flecha, Racionero, Tintoré y Arbós, 20 I4) y además, queremos garantizar que el máximo número de alumnos puedan participar y analizar en profundidad las situaciones que se les presentan en las prácticas en las que están inmersos (Biesta, 20I2).

\section{Metodología}

\section{I. Objetivos}

Los objetivos que en esta investigación nos planteamos, son los siguientes:

- Hacer públicos los dos cuestionarios de evaluación utilizados con los voluntarios universitarios, que hacen referencia a la satisfacción (SAVU-CdA) y de valoración (VAVU-CdA) de las prácticas en CdA.

- Conocer las puntuaciones obtenidas en cada ítem de los cuestionarios mencionados anteriormente.

- Obtener información de los voluntarios universitarios acerca de los aspectos positivos y de los que necesitan mejorar en estas prácticas psicoeducativas en CdA.

\subsection{Participantes}

Los sujetos participantes en las respuestas al cuestionario fueron 100 , de los cuales han contestado el cuestionario de valoración 99 estudiantes, II varones y 88 mujeres y el cuestionario de satisfacción lo han cumplimentado los 100 estudiantes, 12 varones y 88 mujeres. Todos ellos han colaborado durante el curso 2013-14 como voluntarios en las CdA de la provincia de Sevilla. 
La mayoría de ellos son estudiantes de Grado (90\%), aunque hay algunos de las antiguas titulaciones de Diplomatura o Licenciatura, y casi todos $(95 \%)$ de las diferentes titulaciones de la Facultad de Ciencias de la Educación (Pedagogía, Psicopedagogía, Maestros o Actividades Físicas y Deportivas).

Los centros constituidos como CdA en la provincia de Sevilla fueron 12 en el curso 2013-14. Los voluntarios han prestado su colaboración, mayoritariamente, en los situados en la ciudad de Sevilla o en localidades muy próximas y/o bien comunicadas. A los cuatro centros peor comunicados con la ciudad no se apuntó ningún alumno. Todos son Centros de Educación Infantil y Primaria (CEIP) salvo dos, un Instituto de Educación Secundaria (IES) y un Centro de Convenio de Educación Especial (CCEE). Algunos voluntarios habían comenzado su colaboración en CdA en cursos anteriores (27), y por tanto repetían su voluntariado en el curso citado y 72 se incorporaban por primera vez.

La tarea en la que la gran mayoría de los estudiantes colaboraron fue en grupos interactivos $(\mathrm{Gl})$, seguida de las tertulias que se realizaban con adultos, especialmente las tertulias literarias (TLA), en las que participaban también docentes y familiares, $y$ en menor medida en las tertulias pedagógicas (TPA) en las que los participantes eran fundamentalmente docentes. Otras tareas en las que colaboraron fueron la biblioteca tutorizada (BT), tertulias literarias con escolares (TLE), apoyo a los profesores (APO) y en excursiones fuera del centro, apoyo en los recreos y otras (OTR).

\subsection{Instrumentos}

Los instrumentos de evaluación de la actividad fueron dos:

I) Cuestionario de Valoración del Voluntariado Universitario en Comunidades de Aprendizaje (VAVU-CdA).

Se trata de un cuestionario de $10 \mathrm{I}$ ítem agrupados en 3 bloques, cada uno de los cuales se subdivide a su vez en dos sub-bloques como se muestra en la tabla I. 


\begin{tabular}{|c|c|c|c|}
\hline \multicolumn{2}{|c|}{ BLOQUE } & SUB-BLOQUE & ÍTEM \\
\hline \multirow{2}{*}{$\begin{array}{l}\text { Bloque I: } \\
\text { Valoración } \\
\text { de CdA en } \\
\text { general }\end{array}$} & \multicolumn{2}{|c|}{ (I) Aspectos positivos } & $1-12$ \\
\hline & \multicolumn{2}{|c|}{ (II) Aspectos negativos y propuestas de mejora } & $13-24$ \\
\hline \multirow{10}{*}{$\begin{array}{l}\text { Bloque 2: } \\
\text { Valoración } \\
\text { de las } \\
\text { tareas con } \\
\text { escolares } \\
\text { (Grupos } \\
\text { Interactivos } \\
\text { y Biblioteca } \\
\text { tutorizada) }\end{array}$} & \multirow{7}{*}{$\begin{array}{l}\text { (III) } \\
\text { Aspectos } \\
\text { positivos }\end{array}$} & (IIla) Aspectos generales & $25-29$ \\
\hline & & (IIIlb) En relación con la mejora de los escolares & $30-34$ \\
\hline & & (IIlc) En relación con el trabajo cooperativo & $35-39$ \\
\hline & & (IIId) En relación con presencia de familias en aula & $40-44$ \\
\hline & & $\begin{array}{l}\text { (IIle) En relación con presencia de voluntariado en } \\
\text { aula }\end{array}$ & $45-49$ \\
\hline & & (IIIf) En relación con la atención a la diversidad & $50-54$ \\
\hline & & (IIlg) Otros aspectos & $55-58$ \\
\hline & \multirow{3}{*}{$\begin{array}{l}\text { (IV) } \\
\text { Aspectos } \\
\text { negativos y } \\
\text { propuestas } \\
\text { de mejora }\end{array}$} & (IVa) En relación con docentes y voluntariado & $59-63$ \\
\hline & & (IVb) En relación con los escolares & $64-70$ \\
\hline & & (IVc) En relación con la organización del trabajo & $7 I-75$ \\
\hline \multirow{5}{*}{$\begin{array}{l}\text { Bloque 3: } \\
\text { Valoración } \\
\text { de tareas } \\
\text { con adultos } \\
\text { (tertulias } \\
\text { dialógicas) }\end{array}$} & \multirow{4}{*}{$\begin{array}{l}\text { (V) } \\
\text { Aspectos } \\
\text { positivos }\end{array}$} & (Va) Valoraciones generales & $76-80$ \\
\hline & & (Vb) Los temas de diálogo & $81-86$ \\
\hline & & (Vc) Aprendizajes & $87-91$ \\
\hline & & (Vd) Desarrollo profesional, personal y académico & $92-96$ \\
\hline & \multicolumn{2}{|c|}{ (VI) Aspectos negativos y propuestas de mejora } & $97-101$ \\
\hline
\end{tabular}

Tabla I. Estructura del Cuestionario de Valoración del Voluntariado Universitario en Comunidades de Aprendizaje (VAVU-CdA)

2) Cuestionario de Satisfacción del Voluntariado Universitario en Comunidades de Aprendizaje (SAVU-CdA).

Se trata de un cuestionario de 54 ítem agrupados en 3 bloques, cada uno de los cuales se subdividen a su vez en varios sub-bloques como se muestra en la tabla 2. 


\begin{tabular}{|c|c|c|c|}
\hline BLOQUE & & SUB-BLOQUE & ÍTEM \\
\hline \multicolumn{4}{|l|}{$\begin{array}{l}\text { Datos de identificación y } \\
\text { perfil del participante. }\end{array}$} \\
\hline \multirow{2}{*}{$\begin{array}{l}\text { Bloque I: Antes de } \\
\text { comenzar: conocimientos, } \\
\text { motivaciones y expectativas. }\end{array}$} & (I) & Razones por las que decidiste inscribirte en CdA. & $1-6$ \\
\hline & (II) & Expectativas antes de comenzar. & $7-12$ \\
\hline \multirow{2}{*}{$\begin{array}{l}\text { Bloque 2: Durante el desa- } \\
\text { rrollo: percepciones durante } \\
\text { el periodo de la colaboración. }\end{array}$} & (III) & Cómo te has sentido en relación contigo mismo. & $13-18$ \\
\hline & (IV) & Cómo te has sentido en relación con los demás. & $19-24$ \\
\hline \multirow{5}{*}{$\begin{array}{l}\text { Bloque 3: Al terminar: } \\
\text { utilidad de tu colaboración y } \\
\text { valoración global. }\end{array}$} & (V) & $\begin{array}{l}\text { Para qué te ha servido colaborar en } \mathrm{CdA} \text { en el } \\
\text { plano personal. }\end{array}$ & $25-30$ \\
\hline & (VI) & $\begin{array}{l}\text { Para qué te ha servido colaborar en } \mathrm{CdA} \text { en el } \\
\text { plano académico. }\end{array}$ & $31-36$ \\
\hline & (VII) & $\begin{array}{l}\text { Para qué te ha servido colaborar en } \mathrm{CdA} \text { en el } \\
\text { plano profesional. }\end{array}$ & $37-42$ \\
\hline & (VIII) & $\begin{array}{l}\text { Para qué te ha servido colabora en } \mathrm{CdA} \text { en el } \\
\text { plano social. }\end{array}$ & $43-48$ \\
\hline & (IX) & Valoración global de tu participación en CdA. & $49-54$ \\
\hline
\end{tabular}

Tabla 2. Estructura del Cuestionario de Satisfacción del Voluntariado Universitario en Comunidades de Aprendizaje (SAVU-CdA)

Los ítem están formulados como afirmaciones donde hay que señalar el grado de acuerdo o desacuerdo en una escala entre I (nada de acuerdo) y 5 (totalmente de acuerdo). Los dos cuestionarios se presentaron on-line. La fiabilidad de los mismos, descrita en otros trabajos (Aguilera y Gómez del Castillo, 2014) se realizó mediante acuerdo entre jueces. Tres investigadores analizaron independientemente el sistema de categorías y las aportaciones que se incluyeron en cada una de ellas. Las discrepancias se resolvieron mediante consenso. Después se recodificaron las aportaciones aplicando el sistema de categorías creado y se comprobó la fiabilidad intercodificadores.

Para cumplimentar los cuestionarios, esperamos a finalizar el curso, y en los últimos días de mayo los estudiantes colaboradores recibieron un correo electrónico con las direcciones web en las que se encontraban los cuestionarios y las fechas para cumplimentarlos. La aplicación de Google-Drive recogió las respuestas en una hoja de cálculo que fue la base para el procesamiento de los datos. 


\section{Resultados}

\section{A) CUESTIONARIO DE VALORACIÓN DE COMUNIDADES DE APRENDIZAJE}

\section{I. Resultados por bloques generales}

Si atendemos a los resultados considerados por bloques encontramos una mayor puntuación media y una menor dispersión en los aspectos positivos de Comunidades de Aprendizaje en general y del trabajo realizado con escolares (fundamentalmente, grupos interactivos) que en los aspectos negativos de ambos bloques. Ambas cosas hablan positivamente de la valoración que los estudiantes hacen de CdA ya que los datos anteriores significan un mayor acuerdo con los ítem que informan de aspectos positivos y un menor acuerdo con aquellos otros que reflejan elementos negativos de las actuaciones realizadas. No ocurre lo mismo en el caso de la valoración de las tertulias dialógicas en las que las puntuaciones de los aspectos positivos y los negativos están muy próximas.

\begin{tabular}{l|l|c|c}
\cline { 3 - 4 } \multicolumn{2}{l|}{} & MED & DT \\
\hline $\begin{array}{l}\text { Bloque I: Valoración de } \\
\text { CdA en general. }\end{array}$ & (I) Aspectos positivos. & 4,48 & 0,78 \\
\cline { 2 - 5 } & (II) Aspectos negativos y propuestas de mejora. & 3,07 & $\mathrm{I}, 27$ \\
\hline $\begin{array}{l}\text { Bloque 2: Valoración de } \\
\text { las tareas con escolares } \\
\begin{array}{l}\text { (Grupos Interactivos y } \\
\text { Biblioteca tutorizada). }\end{array}\end{array}$ & (III) Aspectos positivos. & 4,36 & 0,83 \\
\cline { 2 - 5 } & (IVpectos negativos y propuestas de mejora. & 2,8 & 2,3 \\
\hline $\begin{array}{l}\text { Bloque 3: Valoración } \\
\text { de tareas con adultos } \\
\text { (tertulias dialógicas). }\end{array}$ & (V) Aspectos positivos. & $3,7 \mathrm{I}$ & $\mathrm{I}, 12$ \\
\cline { 2 - 5 } & (VI) Aspectos negativos y propuestas de mejora. & 3,45 & $\mathrm{I}, 12$ \\
\hline
\end{tabular}

Tabla 3. Media y desviación típica en cada bloque del VAVU-CdA

\subsection{Resultados por ítem}

\subsection{Valoración de Comunidades de Aprendizaje en general. (I) Aspectos positivos}

En once de los doce ítem que forman este sub-bloque se obtiene una puntuación media por encima de 4 puntos. También en once ítem la desviación típica de las puntuaciones es menor de I punto, lo que indica que todas ellas se sitúan muy cerca de la media. 


\begin{tabular}{|c|c|c|c|}
\hline & ÍTEM & MED & DT \\
\hline I) & Mi valoración general de Comunidades de Aprendizaje es positiva. & 4,65 & 0,61 \\
\hline 2) & $\begin{array}{l}\text { En el centro hay muy buen ambiente. Las relaciones entre familias, } \\
\text { docentes y escolares son muy buenas. }\end{array}$ & 4,28 & 0,71 \\
\hline 3) & $\begin{array}{l}\text { Una cosa que destaca en la CdA es el respeto del alumnado hacia los } \\
\text { docentes, hacia el voluntariado y hacia sus compañeros. }\end{array}$ & 4,20 & 0,87 \\
\hline 4) & $\begin{array}{l}\text { Un elemento positivo de CdA es la presencia de las familias en la vida del } \\
\text { colegio. }\end{array}$ & 4,10 & 1,17 \\
\hline 5) & $\begin{array}{l}\text { En CdA no se discrimina a nadie por razón de sexo, edad, origen social, } \\
\text { cultura, etc. }\end{array}$ & 4,74 & 0,54 \\
\hline 6) & $\begin{array}{l}\text { En CdA se trabaja a favor de la igualdad social y educativa de toda la } \\
\text { comunidad. }\end{array}$ & 4,79 & 0,46 \\
\hline 7) & $\begin{array}{l}\text { Una de las cosas más positivas de CdA es que desarrolla la cooperación } \\
\text { frente al individualismo. Se desarrolla el sentido de comunidad. }\end{array}$ & 4,69 & 0,56 \\
\hline 8) & El profesorado trata al alumnado como a sus propios hijos. & 3,88 & 0,92 \\
\hline 9) & Las CdA se basan en las fortalezas y no en las carencias del alumnado. & 4,38 & 0,68 \\
\hline 10) & $\begin{array}{l}\text { En CdA es muy positiva la riqueza cultural que aporta la diversidad y } \\
\text { heterogeneidad de las personas que forman parte de la comunidad } \\
\text { educativa. }\end{array}$ & 4,61 & 0,62 \\
\hline II) & $\begin{array}{l}\text { Las CdA son un referente para otros centros. Habría que extenderlas a } \\
\text { otros centros. }\end{array}$ & 4,77 & 0,57 \\
\hline 12) & $\begin{array}{l}\text { Habría que incrementar la presencia de CdA en la formación } \\
\text { universitaria. }\end{array}$ & 4,68 & 0,64 \\
\hline
\end{tabular}

Tabla 4. Puntuaciones de los ítem del bloque "Aspectos positivos de CdA en general"

Las características de Comunidades de Aprendizaje sobre las que existe un mayor grado de acuerdo y una menor dispersión son: a) que en $\mathrm{CdA}$ se trabaja a favor de la igualdad social y educativa de toda la comunidad ( $M E D=4,79 ; D T=0,46$ ), b) que son un referente para otros centros a los que habría que llevar las actuaciones educativas que se proponen desde $\mathrm{CdA}$ (MED=4,77; $D T=0,57)$, c) que en CdA no se discrimina a nadie por razón de sexo, edad, origen social, cultura, etc. (MED =4,74; $\mathrm{DT}=0,54)$ y d) que en $\mathrm{CdA}$ se desarrolla el sentido de comunidad, la cooperación frente al individualismo $(M E D=4,69 ; D T=0,56)$. 
3.2.2. Valoración de Comunidades de Aprendizaje en general. (II) Aspectos negativos y propuestas de mejora

Las puntuaciones medias en los ítem de este sub-bloque son más bajas que los del anterior y las desviaciones típicas son mayores, lo que significa que la valoración negativa de las $\mathrm{CdA}$ es menos intensa que la valoración positiva y que el grado de acuerdo con las afirmaciones sobre los aspectos negativos es, además mucho menor que el que existe sobre los aspectos positivos. Entre los aspectos negativos y propuestas de mejora que más se destacan son: a) que habría que favorecer más la motivación por seguir estudiando en otras etapas educativas posteriores (MED =3,87; $D T=I, I 2)$, b) que las Comunidades de Aprendizaje exigen mucho trabajo y dedicación al profesorado $(M E D=3,64 ; D T=I, 04), c)$ que habría que informar mejor a los estudiantes universitarios acerca de cómo poder colaborar en $\mathrm{CdA}(\mathrm{MED}=3,40$; $\mathrm{DT}=\mathrm{I}, 29)$ y d) que habría que mejorar la formación de las familias $(M E D=3,39 ; D T=I, I I)$.

\begin{tabular}{|c|c|c|c|}
\hline & ÍTEM & MED & DT \\
\hline & $\begin{array}{l}\text { Comunidades de Aprendizaje exige mucho trabajo y dedicación al } \\
\text { profesorado. }\end{array}$ & 3,64 & 1,04 \\
\hline 14) & Se tiene en cuenta el perfil del voluntario al asignarle una tarea. & 2,81 & 1,32 \\
\hline 15) & Hay niños que necesitan atención individualizada y en CdA no la reciben. & 2,45 & 1,31 \\
\hline 16) & $\begin{array}{l}\text { La información a los estudiantes universitarios es deficiente. Habría que } \\
\text { informar mejor acerca de cómo colaborar en CdA. }\end{array}$ & 3,40 & 1,29 \\
\hline 17) & Habría que mejorar la formación de las familias ya que es escasa. & 3,39 & $\mathrm{I}, \mathrm{II}$ \\
\hline 18) & Habría que mejorar la formación y motivación del profesorado. & 2,85 & 1,23 \\
\hline 19) & La presencia de las familias en el centro es escasa. Habría que incrementarla & 3,09 & 1,36 \\
\hline 20) & $\begin{array}{l}\text { La participación del voluntariado en la CdA es muy escasa. Debería implicar- } \\
\text { se más en el diseño de actividades con niños, en talleres con familias, etc. }\end{array}$ & 2,99 & 1,27 \\
\hline 21) & $\begin{array}{l}\text { La enseñanza de estrategias de resolución de problemas interpersonales y la } \\
\text { mejora de las competencias sociales están poco atendidas en mi centro. }\end{array}$ & 2,20 & 0,95 \\
\hline 22) & Habría que prestar más atención a la orientación vocacional de los escolares. & 3,03 & 1,10 \\
\hline 23) & Habría que incrementar el aprendizaje de materias instrumentales. & 3,13 & 1,17 \\
\hline 24) & $\begin{array}{l}\text { Habría que favorecer más la motivación por seguir estudiando en otras } \\
\text { etapas educativas posteriores. }\end{array}$ & 3,87 & 1,12 \\
\hline
\end{tabular}

Tabla 5. Puntuaciones de los ítem del bloque "Aspectos negativos de CdA en general" 


\subsubsection{Valoración de las tareas con escolares. (I) Aspectos positivos}

Dentro de este apartado vamos a considerar aspectos positivos relacionados con las tareas realizadas con escolares. Recordemos que estas tareas se refieren fundamentalmente a Grupos Interactivos y en menor medida a actuaciones en la Biblioteca Tutorizada.

\subsection{3.a) Aspectos positivos relacionados con cuestiones generales}

Todos los ítem de este bloque obtienen una puntuación superior a 4 (media de 4,38 ) y una desviación típica inferior a I, lo que indica un alto grado de acuerdo con los aspectos positivos de $\mathrm{CdA}$ a los que ellos se refieren.

\begin{tabular}{l|c|c}
\hline \multicolumn{1}{c|}{ ÍTEM } & MED & DT \\
\hline $\begin{array}{l}\text { 25) La valoración general de la organización del trabajo con el alumnado es } \\
\text { positiva. }\end{array}$ & 4,34 & 0,70 \\
\hline 26) El trabajo en grupos interactivos deberían extenderse a otros centros. & 4,87 & 0,40 \\
\hline $\begin{array}{l}\text { 27) Los chicos/as se adaptan pronto al trabajo en equipos donde dialogan y se } \\
\text { ayudan. }\end{array}$ & 4,29 & 0,82 \\
\hline $\begin{array}{l}\text { 28) El clima de trabajo es adecuado para un buen aprendizaje de las materias } \\
\text { escolares. }\end{array}$ & 4,14 & 0,91 \\
\hline 29) El clima de trabajo es emocionalmente gratificante. & 4,25 & 0,88 \\
\hline
\end{tabular}

Tabla 6. Puntuaciones de los ítem "Aspectos positivos de los grupos interactivos en general"

\subsection{3.b) Aspectos positivos relacionados con la mejora de los escolares}

En los cinco ítem referidos a aspectos relacionados con la mejora de los estudiantes, la media de las puntuaciones es de 4,2 y las desviaciones típicas se sitúan entre 0,66 y 0,84 . Es decir, el acuerdo con estos aspectos positivos obtenido por el conjunto de estudiantes universitarios voluntarios es muy alto. 


\begin{tabular}{l|c|c}
\hline \multicolumn{1}{c|}{ ÍTEM } & MED & DT \\
\hline $\begin{array}{l}\text { 30) Con los grupos interactivos mejora el rendimiento de los escolares y se } \\
\text { acelera el aprendizaje. }\end{array}$ & 4,22 & 0,7 I \\
\hline 31) Con los grupos interactivos los niños mantienen más la atención. & 4,02 & 0,84 \\
\hline $\begin{array}{l}\text { 32) Con grupos interactivos los escolares trabajan más y mejor. Se está } \\
\text { aprendiendo durante toda la jornada. }\end{array}$ & 4,15 & 0,79 \\
\hline 33) Con los grupos interactivos se incrementa la motivación de los escolares. & 4,34 & 0,66 \\
\hline $\begin{array}{l}\text { 34) El trabajo en grupos interactivos mejora la valoración que cada chico/a } \\
\text { hace de sí mismo y de sus compañeros/as. }\end{array}$ & 4,20 & 0,74 \\
\hline
\end{tabular}

Tabla 7. Puntuaciones de los ítem

"Aspectos positivos de los grupos interactivos en relación con la mejora de los escolares"

\subsection{3.c) Aspectos positivos relacionados con el trabajo cooperativo}

En este apartado el grado de acuerdo es muy alto a juzgar por la puntuación media obtenida $(4,5)$. Y por otra parte la dispersión de las puntuaciones en torno a cada media también es muy baja variando entre 0,59 y 0,72 .

\begin{tabular}{l|c|c}
\hline \multicolumn{1}{c|}{ ÍTEM } & MED & DT \\
\hline $\begin{array}{l}\text { 35) Un aspecto positivo de los grupos interactivos es la relación de ayuda que } \\
\text { se establece entre compañeros. }\end{array}$ & 4,43 & 0,67 \\
\hline $\begin{array}{l}\text { 36) Con los grupos interactivos los escolares mejoran en comportamientos y } \\
\text { habilidades sociales. }\end{array}$ & 4,42 & 0,63 \\
\hline 37) En la relación de ayuda se beneficia tanto la persona que ayuda como la ayudada. & 4,6 I & 0,59 \\
\hline 38) La presencia de familias y voluntarios facilita la cooperación entre escolares. & 4,48 & 0,72 \\
\hline 39) El trabajo cooperativo permite trabajar con grupos heterogéneos de escolares. & 4,58 & 0,61 \\
\hline
\end{tabular}

Tabla 8. Puntuaciones de los ítem

"Aspectos positivos de los grupos interactivos en relación con el trabajo cooperativo"

\subsection{3.d) Aspectos positivos relacionados con la presencia de familias en el aula}

La presencia de las familias en el aula es también un aspecto muy valorado. Las puntuaciones medias que indican el grado de acuerdo con cada uno de los cinco ítem 
que componen este sub-apartado se encuentran entre 4, 19 y 4,47 y las desviaciones típicas entre 0,86 y 0,93 .

\begin{tabular}{l|c|c}
\hline \multicolumn{1}{c|}{ ÍTEM } & MED & DT \\
\hline \begin{tabular}{l} 
40) $\begin{array}{l}\text { Con la presencia de familias en las aulas se fortalece el vínculo entre } \\
\text { familias y escuela. }\end{array}$ \\
\hline 4I) La presencia de familiares en las aulas mejora las relaciones familiares.
\end{tabular} & 4,29 & 0,86 \\
\hline $\begin{array}{l}\text { 42) Los grupos interactivos incrementan y facilitan la presencia de familias en } \\
\text { el centro. }\end{array}$ & 4,19 & 0,92 \\
\hline $\begin{array}{l}\text { 43) La presencia de familiares y voluntarios aumentan las perspectivas de los } \\
\text { escolares. }\end{array}$ & 4,24 & 0,93 \\
\hline 44) La presencia de familias en el aula refuerza la autoestima positiva en los niños. & 4,30 & 0,91 \\
\hline
\end{tabular}

Tabla 9. Puntuaciones de los ítem

"Aspectos positivos de los grupos interactivos en relación con la presencia de las familias en el aula"

\subsection{3.e) Aspectos positivos relacionados con la presencia de voluntariado en el aula}

Continuando con lo dicho anteriormente respecto a la presencia de las familias en el aula, las aportaciones referidas a la presencia del voluntariado en general van en la misma línea, a excepción del ítem 46, donde existen voluntarios que sí ven problema en que los adultos desconozcan los contenidos a enseñar.

\begin{tabular}{l|c|c}
\hline \multicolumn{1}{c|}{ ÍTEM } & MED & DT \\
\hline 45) La presencia de otros adultos en el aula permite atender mejor a los chicos. & 4,58 & 0,62 \\
\hline $\begin{array}{l}\text { 46) El hecho de que algunos adultos voluntarios desconozcan los contenidos } \\
\text { a enseñar no es un problema. }\end{array}$ & 3,02 & 1,23 \\
\hline $\begin{array}{l}\text { 47) Los docentes y el voluntariado se adaptan rápidamente al trabajo en } \\
\text { grupos interactivos. }\end{array}$ & 4,48 & 0,66 \\
\hline $\begin{array}{l}\text { 48) La imagen de los voluntarios jóvenes y su presencia en el aula es un } \\
\text { estímulo para los escolares. }\end{array}$ & 4,67 & 0,55 \\
\hline 49) Se da un aprendizaje intergeneracional y con los iguales. & 4,48 & 0,66 \\
\hline
\end{tabular}

Tabla 10. Puntuaciones de los ítem "Aspectos positivos de los grupos interactivos en relación con presencia en el aula del voluntariado en general" 


\subsection{3.f) Aspectos positivos relacionados con la atención a la diversidad}

Existe un alto grado de acuerdo con el hecho de que las actuaciones educativas que se proponen desde Comunidades de Aprendizaje favorecen la atención a la diversidad, superando en todos los ítem el cuatro de media y la desviación típica no supera el 0,8 en ninguno de ellos.

\begin{tabular}{l|c|c}
\hline \multicolumn{1}{c|}{ ÍTEM } & MED & DT \\
\hline 50) En grupos interactivos se fomenta la superación de desigualdades. & 4,52 & 0,63 \\
\hline 5I) En grupos interactivos se atiende a la diversidad cultural. & 4,63 & 0,65 \\
\hline 52) Los grupos interactivos permiten combinar un seguimiento individual y grupal. & 4,44 & 0,73 \\
\hline $\begin{array}{l}\text { 53) En los grupos interactivos el alumnado desempeña un papel activo en su } \\
\text { proceso de aprendizaje }\end{array}$ & 4,49 & 0,72 \\
\hline $\begin{array}{l}\text { 54) En el trabajo con los escolares se cuenta con la opinión de ellos mediante } \\
\text { un diálogo igualitario. }\end{array}$ & 4,22 & 0,74 \\
\hline
\end{tabular}

Tabla II. Puntuaciones de los ítem "Aspectos positivos de los grupos interactivos en relación con la atención a la diversidad"

\subsection{3.g) Aspectos positivos relacionados con otros aspectos}

Otros aspectos positivos que también se destacan son los relacionados con las tareas que desarrollan los escolares, como que los voluntarios también aprenden $(M E D=4,83 ; D T=0,50)$, que reciben ayuda cada vez que lo necesitan ( $M E D=4,59$; $\mathrm{DT}=0,6 \mathrm{I})$ y que los grupos interactivos atrapan la atención y el interés de los voluntarios ("te enganchan") (MED $=4,42 ; \mathrm{DT}=0,85)$.

\begin{tabular}{l|c|c}
\hline \multicolumn{1}{c|}{ ÍTEM } & MED & DT \\
\hline 55) Los voluntarios también aprendemos. & 4,83 & 0,50 \\
\hline 56) El voluntariado recibe del docente la ayuda que necesita para hacer su tarea. & 4,59 & $0,6 \mathrm{I}$ \\
\hline $\begin{array}{l}\text { 57) Los grupos interactivos te enganchan. } \\
\begin{array}{l}\text { 58) Se cuenta con la opinión del voluntariado a la hora de asignarle curso y } \\
\text { horario. }\end{array}\end{array}$ & 4,42 & 0,85 \\
\hline
\end{tabular}

Tabla 12. Puntuaciones de los ítem

"Aspectos positivos de los grupos interactivos en relación con otros aspectos" 
3.2.4. Valoración de las tareas con escolares. (II) Aspectos negativos y propuestas de mejora

El grado de acuerdo con los ítem que se refieren a aspectos negativos es menor que el obtenido en relación con los aspectos positivos. También es mayor la dispersión de las puntuaciones en los aspectos negativos que en los positivos, lo que indica un menor grado de coincidencia en la valoración que de ellos hace el conjunto de personas que han respondido al cuestionario.

No obstante, es clave atender a estas valoraciones de los estudiantes con el fin de seguir mejorando la propuesta de voluntariado que se les hace así como el desarrollo de las actuaciones educativas que se llevan a cabo en el marco de las CdA.

\subsection{4.a) Aspectos negativos relacionados con docentes y voluntariado}

Entre los aspectos a mejorar que más se destacan (con una puntuación cercana a la de "bastante de acuerdo") en relación con la presencia de adultos en el aula se encuentra el de la necesidad de aumentar el número de voluntarios, especialmente fomentando más la participación de familias y personas del barrio en el que se encuentra el centro escolar $(M E D=3,89$; $D T=0,99)$, en segundo lugar, con puntuaciones en torno a 3 puntos (suficientemente de acuerdo) se destacan la necesidad de dar más protagonismo e iniciativa al voluntariado (MED=3, I4; $\mathrm{DT}=1,08)$ y mejorar la coordinación entre docentes y voluntariado (MED $=2,92$; $\mathrm{DT}=\mathrm{I}, 23)$.

\begin{tabular}{l|c|c}
\hline \multicolumn{1}{c|}{ ÍTEM } & MED & DT \\
\hline $\begin{array}{l}\text { 59) El comportamiento de algunos docentes deja mucho que desear. No se } \\
\text { ajusta a los principios dialógicos que subyacen a CdA. }\end{array}$ & 2,17 & $\mathrm{I}, 17$ \\
\hline 60) Es necesario mejorar la coordinación entre voluntariado y profesorado. & 2,92 & $\mathrm{I}, 23$ \\
\hline 6I) Habría que dar más protagonismo e iniciativa a los voluntarios/as. & 3,14 & $\mathrm{I}, 08$ \\
\hline $\begin{array}{l}\text { 62) La falta de asistencia de los voluntarios sin avisar ha sido un gran } \\
\text { problema. }\end{array}$ & 2,93 & $\mathrm{I}, 38$ \\
\hline $\begin{array}{l}\text { 63) Es necesario aumentar el número de voluntarios y voluntarias } \\
\text { fomentando la participación de familiares y personas del barrio. }\end{array}$ & 3,89 & 0,99 \\
\hline
\end{tabular}

Tabla 13. Puntuaciones de los ítem "Aspectos negativos y propuestas de mejora de los grupos interactivos en relación con los docentes y el voluntariado" 


\subsection{4.b) Aspectos negativos relacionados con los escolares}

Solo con un grado de acuerdo cercano a 4 (bastante de acuerdo) se señala la necesidad de fomentar más la presencia de los escolares en la biblioteca tutorizada $(M E D=3,8 \mathrm{I} ; \mathrm{DT}=\mathrm{I}, 06)$. En los demás item, como se observa en la siguiente tabla, se puntúa por debajo de 3,3 de media.

\begin{tabular}{l|c|c}
\hline \multicolumn{1}{c|}{ ÍTEM } & MED & DT \\
\hline 64) Trabajar en grupos interactivos con los niños pequeños es difícil. & $2,2 \mathrm{I}$ & $\mathrm{I}, 08$ \\
\hline 65) Ha habido poca asistencia de niños a la biblioteca tutorizada. & 2,76 & $\mathrm{I}, 07$ \\
\hline 66) Habría que fomentar la asistencia del alumnado a la biblioteca tutorizada. & $3,8 \mathrm{I}$ & $\mathrm{I}, 06$ \\
\hline 67) Los niños se comportan mal en el centro. & 2,29 & 0,97 \\
\hline $\begin{array}{l}\text { 68) Los niños de la misma nacionalidad, cuando pueden, hacen grupitos y se } \\
\text { separan de los niños de las otras nacionalidades. }\end{array}$ & 1,67 & 0,93 \\
\hline $\begin{array}{l}\text { 69) Habría que fomentar más las relaciones entre alumnos de distintos cursos } \\
\text { o nacionalidades. }\end{array}$ & 3,22 & 1,27 \\
\hline $\begin{array}{l}\text { 70) En los grupos interactivos, los chicos/as que van peor retrasan a los que } \\
\text { van mejor. }\end{array}$ & 2,10 & 1,04 \\
\hline
\end{tabular}

Tabla 14. Puntuaciones de los ítem "Aspectos negativos y propuestas de mejora de los grupos interactivos en relación con los escolares"

\subsection{4.c) Aspectos negativos relacionados con la organización del trabajo en el aula}

En cuanto a la organización del trabajo en el aula, hay dos aspectos que destacan porque se está suficientemente de acuerdo con ellos (puntuaciones en torno a 3). Son los siguientes: a) hay que introducir actividades y metodologías más lúdicas $(M E D=3,32 ; D T=I, 16), y$ b) diversificar más las actividades de la biblioteca tutorizada $(M E D=2,94 ; D T=I, 00)$. Globalmente no se percibe que en la organización del trabajo en el aula se contemple que haya niños y niñas que realicen tareas diferentes a las del resto del grupo $(M E D=I, 79 ; D T=I, I 8)$. 


\begin{tabular}{l|c|c}
\hline \multicolumn{1}{c|}{ ÍTEM } & MED & DT \\
\hline 7I) Se hacían grupos interactivos siempre que estaban planificados. & 3,72 & I, I8 \\
\hline 72) Algunos niños hacían tareas diferentes a los demás. & 1,79 & I, 18 \\
\hline 73) Es necesario introducir metodologías y actividades más lúdicas. & 3,32 & $\mathrm{I}, 16$ \\
\hline 74) Habría que diversificar las actividades de la biblioteca tutorizada. & 2,94 & $\mathrm{I}, 00$ \\
\hline 75) Hay una gran discontinuidad entre los contextos familiar y escolar. & 2,95 & $\mathrm{I}, 19$ \\
\hline
\end{tabular}

Tabla 15. Puntuaciones de los ítem "Aspectos negativos y propuestas de mejora de los grupos interactivos en relación con la organización del trabajo"

\subsubsection{Valoración de las tareas con adultos. (I) Aspectos positivos}

Como hemos dicho anteriormente, las tareas realizadas con adultos en las Comunidades de Aprendizaje han sido fundamentalmente tertulias dialógicas, bien literarias, bien pedagógicas. A continuación entraremos en detalle sobre los aspectos positivos encontrados en ellas agrupados en cuatro secciones:

\subsection{5.a) Aspectos positivos relacionados con valoraciones generales}

De las tertulias literarias se destaca una valoración general positiva (bastante de acuerdo) ( $M E D=4,05 ; D T=0,96)$, especialmente el buen ambiente que se crea en ellas $(M E D=4,13$; $D T=0,95)$.

\begin{tabular}{l|c|c}
\hline \multicolumn{1}{c|}{ ÍTEM } & MED & DT \\
\hline 76) La valoración general de las tertulias es positiva. & 4,05 & 0,96 \\
\hline $\begin{array}{l}\text { 77) Uno de los aspectos mejores de las tertulias es el buen ambiente que se } \\
\text { crea. }\end{array}$ & 4,13 & 0,95 \\
\hline 78 En las tertulias se implican todos los participantes. & 3,44 & $\mathrm{I}, 13$ \\
\hline 79) Las tertulias contribuyen a la promoción de las familias. & 3,65 & $\mathrm{I}, 14$ \\
\hline $\begin{array}{l}\text { 80) Me han permitido observar y/o practicar lo que he aprendido en la } \\
\text { facultad. }\end{array}$ & 3,32 & $\mathrm{I}, 19$ \\
\hline
\end{tabular}

Tabla 16. Puntuaciones de los ítem "Aspectos positivos de las tertulias dialógicas, literarias y pedagógicas, en relación con aspectos generales" 


\subsection{5. b) Aspectos positivos relacionados con los temas de diálogo}

Aunque con una alta dispersión en las puntuaciones, los aspectos más destacado en relación con los diálogos suscitados en las tertulias (puntuaciones en torno a "bastante de acuerdo") son que te permiten conocer la vida de personas diferentes y eso te abre a nuevas realidades $(M R D=4,06 ; D T=I, 0 I)$, que en las tertulias se habla y se reflexiona sobre la vida ya que ayudan a relacionar lo que se lee con lo que se vive $(M E D=3,95$; $D T=0,94)$ y que en las tertulias en las que intervienen familias se rompen prejuicios y estereotipos (MED $=3,89$; $D T=I, 02$ ).

\begin{tabular}{|c|c|c|c|}
\hline & ÍTEM & MED & DT \\
\hline & $\begin{array}{l}\text { En las tertulias se habla y se reflexiona sobre la vida; ayudan a relacionar } \\
\text { lo que lees con la vida. }\end{array}$ & 3,95 & 0,94 \\
\hline 82) & $\begin{array}{l}\text { Las tertulias te permiten conocer la vida de gente diferente y te abren a } \\
\text { otras realidades. }\end{array}$ & 4,06 & $1,0 \mathrm{I}$ \\
\hline 83) & En las tertulias con las familias se rompen estereotipos y prejuicios. & 3,89 & 1,02 \\
\hline 84) & En las tertulias se habla de los hijos y eso ayuda a mejorar su educación. & 3,54 & 1,07 \\
\hline 85) & $\begin{array}{l}\text { Se logra un nivel de comunicación poco habitual en otros contextos que } \\
\text { frecuento. }\end{array}$ & 3,72 & 1,06 \\
\hline 86) & $\begin{array}{l}\text { He dicho cosas y he hecho reflexiones que no había compartido antes } \\
\text { con nadie. }\end{array}$ & 3,24 & 1,23 \\
\hline
\end{tabular}

Tabla 17. Puntuaciones de los ítem "Aspectos positivos de las tertulias dialógicas, literarias y pedagógicas, en relación con los temas de diálogo"

\subsection{5.c) Aspectos positivos relacionados con los aprendizajes realizados}

En general, el grado de acuerdo en torno a los ítem que conforman este subapartado no es tan alto como en otras valoraciones. 


\begin{tabular}{l|c|c}
\hline \multicolumn{1}{c|}{ ÍTEM } & MED & DT \\
\hline 87) Las tertulias han sido un estimulo para leer, despertándome interés por la lectura. & 3,36 & $\mathrm{I}, 04$ \\
\hline $\begin{array}{l}\text { 88) Al preparar las tertulias lees de una forma distinta de cuando lees para ti } \\
\text { solamente. }\end{array}$ & 3,69 & $\mathrm{I}, \mathrm{II}$ \\
\hline 89) En las tertulias se aprende de los demás contertulios. & 4,19 & $0,9 \mathrm{I}$ \\
\hline 90) En las tertulias se aprende a escuchar y compartir reflexiones y opiniones. & 4,32 & 0,97 \\
\hline $\begin{array}{l}\text { 91) Las tertulias ayudan a descentrarte de tu punto de vista y comprender el } \\
\text { del otro aunque discrepes. Aprendes a respetar la opinión de otros. }\end{array}$ & 4,22 & 0,93 \\
\hline
\end{tabular}

Tabla 18. Puntuaciones de los ítem "Aspectos positivos de las tertulias dialógicas, literarias y pedagógicas, en relación con los aprendizajes realizados"

\subsection{5.d) Aspectos positivos relacionados con el desarrollo profesional y académico}

El grado de acuerdo con los aspectos señalados en este sub-apartado se encuentra entre 3 (suficientemente de acuerdo) y 4 (bastante de acuerdo). No obstante, la dispersión en las valoraciones se pone de manifiesto en las desviaciones típicas que en todos los casos son superiores a I punto.

\begin{tabular}{l|c|c}
\hline \multicolumn{1}{c|}{ ÍTEM } & MED & DT \\
\hline 92) Las tertulias me han ayudado en mi futuro trabajo profesional. & 3,53 & $\mathrm{I}, 18$ \\
\hline 93) Me han permitido conocer ámbitos profesionales distintos. & 3,65 & $\mathrm{I}, 24$ \\
\hline 94) En las tertulias he aclarado mi vocación profesional. & 3,16 & $\mathrm{I}, 23$ \\
\hline 95) Las tertulias han sido valiosas para mi desarrollo como persona. & 3,63 & $\mathrm{I}, \mathrm{I5}$ \\
\hline 96) Las tertulias me han ayudado en mis estudios y tareas académicas actuales. & 3,02 & $\mathrm{I}, 24$ \\
\hline
\end{tabular}

Tabla 19. Puntuaciones de los ítem "Aspectos positivos de las tertulias dialógicas, literarias y pedagógicas, en relación con el desarrollo profesional, personal y académico"

\subsubsection{Valoración de las tareas con adultos. (II) Aspectos negativos}

Las valoraciones están cercanas al "bastante de acuerdo" $(3,45)$, en que a las tertulias asisten pocos familiares y que habría que incentivar más su participación $(\mathrm{MED}=3,80 ; \mathrm{DT}=0,98)$, aunque el número total de participantes es adecuado para facilitar una buena comunicación y el diálogo entre todos ( $M E D=3,72$; $D T=0,92)$. 


\begin{tabular}{l|c|c}
\hline \multicolumn{1}{c|}{ ÍTEM } & MED & DT \\
\hline 97) Hay poca asistencia de familiares en las tertulias. Habría que incentivarla más. & 3,80 & 0,98 \\
\hline 98) La participación del voluntariado en las tertulias es pobre y escasa. & 2,98 & 1,18 \\
\hline 99) Las tertulias deberían empezar antes, desde comienzo del curso. & 3,15 & 1,24 \\
\hline I00) Los libros elegidos son adecuados. & 3,58 & 1,00 \\
\hline I0I) El número de participantes es el adecuado para una buena comunicación. & 3,72 & 0,92 \\
\hline
\end{tabular}

\section{B) CUESTIONARIO DE SATISFACCIÓN DE COMUNIDADES DE APRENDIZAJE}

A juzgar por las respuestas dadas en este cuestionario, el grado de satisfacción de los estudiantes universitarios con su colaboración en $\mathrm{CdA}$ es bastante alto siendo la media de todas las respuestas de $4, \mathrm{II}(\mathrm{DT}=\mathrm{I}, \mathrm{I} \mathrm{I})$.

Si analizamos los datos por los sub-bloques, vemos que la puntuación más alta corresponde al de "valoración global de tu participación en CdA" que es de 4,55 (DT=0,82), mayor incluso que la puntuación media obtenida a partir de las respuestas a todos los ítem por parte de todos los participantes.

\begin{tabular}{ll|c|c}
\hline \multicolumn{1}{c|}{ SUB-BLOQUE } & MED & DT \\
\hline (I) & Razones por las que decidiste inscribirte en CdA. & 3,90 & I,35 \\
\hline (II) $\quad$ Expectativas antes de comenzar. & 3,25 & I,59 \\
\hline (III) $\quad$ Cómo te has sentido en relación contigo mismo. & 4,27 & 0,83 \\
\hline (IV) $\quad$ Cómo te has sentido en relación con los demás. & 4,36 & 0,86 \\
\hline (V) $\quad$ Para qué te ha servido colaborar en CdA en el plano personal. & 4,07 & 0,97 \\
\hline (VI) $\quad$ Para qué te ha servido colaborar en CdA en el plano académico. & 4,00 & 1,06 \\
\hline (VII) $\quad$ Para qué te ha servido colaborar en CdA en el plano profesional. & 4,26 & 0,88 \\
\hline (VIII) Para qué te ha servido colabora en CdA en el plano social. & 4,37 & 0,83 \\
\hline (IX) Valoración global de tu participación en CdA. & 4,55 & 0,82 \\
\hline
\end{tabular}

Tabla 2I. Puntuaciones obtenidas en cada bloque de ítem del SAVU-CdA 
A continuación las puntuaciones destacan que los estudiantes valoran la utilidad de su colaboración en $\mathrm{CdA}$ como un medio de tomar conciencia de las cuestiones sociales ( $M E D=4,37 ; D T=0,83$ ), seguido de la utilidad para su formación como profesionales (MED $=4,26$; $D T=0,88$ ), para su crecimiento personal (MED =4,07; $\mathrm{DT}=0,97)$ y para su desarrollo académico $(M E D=4 ; D T=I, 06)$.

\subsection{Bloque I: Antes de comenzar: motivaciones y expectativas}

\section{(I) Motivaciones}

En cuanto a las motivaciones de los estudiantes para colaborar como voluntarios y voluntarias en $\mathrm{CdA}$ destacan tres: realizar más prácticas relacionadas con la educación ( $M E D=4,77 ; D T=0,58)$, trabajar con niños ( $M E D=4,68 ; D T=0,65$ ) y realizar aprendizajes sobre educación que no se imparten en las facultades $(\mathrm{MED}=4,63$; DT=0,58).

\begin{tabular}{ll|c|c}
\hline \multicolumn{1}{c|}{ ÍTEM } & MED & DT \\
\hline I) & $\begin{array}{l}\text { Porque creo que es importante tener más prácticas reales en tareas } \\
\text { relacionadas con la educación. }\end{array}$ & 4,77 & 0,58 \\
\hline 2) & $\begin{array}{l}\text { Porque es una ocasión para aprender teorías y prácticas educativas que } \\
\text { no se enseñan en la facultad: aprendizaje dialógico, comunidades de } \\
\text { aprendizaje,... }\end{array}$ & 4,63 & 0,58 \\
\hline 3) & $\begin{array}{l}\text { Porque tenía experiencia previa de voluntariado o conocía ya el centro o } \\
\text { el barrio y deseaba seguir colaborando en el mismo. }\end{array}$ & 2,45 & 1,5 I \\
\hline 4) Por sensibilidad ante las situaciones de pobreza y marginación. & 3,8 I & 1,04 \\
\hline 5) Porque me gusta trabajar con niños. & 4,68 & 0,65 \\
\hline 6) Para conseguir créditos de Libre Configuración o reconocimiento de & 3,07 & 1,33 \\
\hline
\end{tabular}

Tabla 22. Razones para colaborar como voluntario en Comunidades de Aprendizaje

\section{(II) Expectativas}

En coherencia con los resultados anteriores referidos a las razones para inscribirse como voluntario o voluntaria en Comunidades de Aprendizaje, las expectativas previas destacan que esperan realizar una actividad que le resulte útil para su 
futuro profesional ( $M E D=4,67 ; D T=0,60)$, que sea enriquecedora en lo personal $(M E D=4,64 ; D T=0,64)$ y que le aporte conocimientos relevantes para su actividad académica $(\mathrm{MED}=4,28 ; \mathrm{DT}=0.91)$.

\begin{tabular}{|c|c|c|c|}
\hline & ÍTEM & MED & DT \\
\hline 7) & Tenía pocas expectativas. & 2,00 & 1,16 \\
\hline 8) & $\begin{array}{l}\text { Pensaba que lo que aprendería me resultaría muy útil académicamente en } \\
\text { mis estudios actuales. }\end{array}$ & 4,28 & 0,91 \\
\hline 9) & $\begin{array}{l}\text { Pensaba que lo que podía aprender me sería muy útil en mi futuro } \\
\text { profesional. }\end{array}$ & 4,67 & 0,60 \\
\hline 10) & $\begin{array}{l}\text { Esperaba que fuese una experiencia enriquecedora en lo personal gracias } \\
\text { a las aportaciones de otras personas y abrirme a otras experiencias y } \\
\text { opiniones. }\end{array}$ & 4,64 & 0,64 \\
\hline II) & $\begin{array}{l}\text { Tenía algunos prejuicios acerca del tipo de personas con las que iba } \\
\text { a trabajar y esperaba problemas con los escolares, sus familias, el } \\
\text { entorno,... }\end{array}$ & 1,93 & 1,08 \\
\hline 12) & Pensaba que serían unas prácticas como tantas otras. & 1,96 & 1,04 \\
\hline
\end{tabular}

Tabla 23. Expectativas ante la colaboración en Comunidades de Aprendizaje

Debemos estar atentos a la interpretación de los tres ítem restantes de este apartado (ítem 7, I I y I2) ya que aparecen redactados en forma negativa. Así de los resultados que se muestra en la tabla 23 parece deducirse que una gran mayoría de estudiantes tenían altas expectativas (ítem 7) y no consideraban que su colaboración en CdA fuesen unas prácticas como otras tantas que podrían hacerse (ítem 12). Por otra parte, también eran pocos los participantes que se acercaban con prejuicios acerca de la población con la que iban a trabajar.

\subsection{Bloque 2: Durante el desarrollo: percepciones durante el periodo de la colaboración}

(III) En relación consigo mismo

Todos los ítem de este apartado han obtenido una alta valoración destacando el hecho de que los estudiantes universitarios voluntarios se han sentido orgullosos de 
sí mismo por lo que han sido capaces de hacer $(M E D=4,40$; $D T=0,67)$, percibían que estas prácticas les han hecho crecer y madurar (MED $=4,37$; $D T=0,82)$ y se han sentido realizados en lo profesional (MED $=4,23$; $D T=0,83$ ) hasta el punto de que en algunos momentos llegaban a emocionarse con la tarea que desarrollaban (MED $=4,34$; $\mathrm{DT}=0,86$ ).

Aunque con una media baja, casi tres cuartas partes de los participantes estaban muy de acuerdo o totalmente de acuerdo con la afirmación "me he sentido seguro/a en lo que hacía y cómo lo hacía" (MED=3,92; $D T=0,80$ ).

\begin{tabular}{l|c|c}
\hline \multicolumn{1}{c|}{ ÍTEM } & MED & DT \\
\hline $\begin{array}{l}\text { I3) Me he sentido orgulloso/a de mí mismo/a por lo que he sido capaz de } \\
\text { hacer. }\end{array}$ & 4,40 & 0,67 \\
\hline I4) Me he sentido autorrealizado/a profesionalmente. & 4,23 & 0,83 \\
\hline I5) Me ha hecho crecer y desarrollarme como persona & 4,37 & 0,82 \\
\hline I6) Ha habido momentos en los que me he sentido emocionada/o. & 4,34 & 0,86 \\
\hline I7) Me he sentido seguro/a en lo que hacía y cómo lo hacía. & 3,92 & 0,80 \\
\hline $\begin{array}{l}\text { I8) Sentía que no quería que terminase la sesión. Cuando estaba en el colegio } \\
\text { el tiempo pasaba volando. }\end{array}$ & 4,34 & 0,93 \\
\hline
\end{tabular}

Tabla 24. Percepciones en relación consigo mismo.

(IV) En las relaciones con los demás

También son muy positivamente valoradas las percepciones de los participantes en cuanto a las relaciones con otros miembros de la comunidad educativa. Destaca en ellos la actitud de agradecimiento por la oportunidad que esta colaboración les ha brindado ( $M E D=4,63 ; D T=0,7 \mathrm{I}$ ). Se han visto como en familia, muy bien acogidos por la comunidad educativa que los recibía $(M E D=4,44 ; D T=0,87)$ y al mismo tiempo se han sentido útiles para los demás ( $M E D=4,37 ; D T=0,8 \mathrm{I}$ ) y con libertad para actuar y para aportar sugerencias y puntos de vista (MED $=4,44 ; D T=0,87)$. Igualmente, destacan que su trabajo ha sido reconocido y valorado (MED $=4,20$; $\mathrm{DT}=0,86)$. 


\begin{tabular}{l|c|c}
\hline \multicolumn{1}{c|}{ ÍTEM } & MED & DT \\
\hline 19) Me he sentido útil para los docentes, para los escolares y para el centro. & 4,37 & 0,8 I \\
\hline $\begin{array}{l}\text { 20) Me he sentido agradecido/a por la oportunidad que se me ha ofrecido de } \\
\text { participar. }\end{array}$ & 4,63 & 0,7 I \\
\hline 21) Me he sentido recompensado/a por mi trabajo. & 4,20 & 0,86 \\
\hline 22) Me he sentido con libertad para aportar mi opinión y mi punto vista. & 4,3 I & 0,90 \\
\hline 23) Me he sentido cómodo/a, acogido/a, como en familia. & 4,44 & 0,87 \\
\hline $\begin{array}{l}\text { 24) Me he sentido sorprendido/a por la realidad escolar que me he } \\
\text { encontrado e impresionado/a por la vida de algunas familias. }\end{array}$ & 4,18 & 0,96 \\
\hline
\end{tabular}

Tabla 25. Percepciones en relación con los demás

\subsection{Bloque 3: Al terminar: utilidad de tu colaboración y valoración global}

\section{(V) Utilidad en el plano personal}

Las puntuaciones obtenidas son considerablemente altas en casi todos los ítem superando los cuatro puntos en cuatro de ellos: aprendizaje de valores de solidaridad, respeto y colaboración ( $M E D=4,4 \mathrm{I} ; \mathrm{DT}=0,8 \mathrm{I}$ ) y maduración al tener que enfrentarse a dificultades ( $M E D=4,25 ; D T=0,80$ ), mejora en habilidades sociales y comunicativas $(M E D=4,19 ; D T=0,84)$. Por otra parte, también destacan que han aprendido a valorar más las oportunidades que han tenido en la vida $(M E D=4, I 4$; $D T=0,90)$.

\begin{tabular}{|c|c|c|c|}
\hline & ÍTEM & MED & DT \\
\hline & $\begin{array}{l}\text { Participar en CdA ha sido de gran ayuda en lo personal. Noto que he } \\
\text { madurado más y he aprendido con las dificultades. }\end{array}$ & 4,25 & 0,80 \\
\hline 26) & $\begin{array}{l}\text { Esta experiencia en CdA me ha servido para superar algunos prejuicios } \\
\text { que tenía acerca de la población con la que he trabajado. }\end{array}$ & 3,65 & 1,23 \\
\hline 27) & Tras mi aportación en CdA ha mejorado mi autoestima. & 3,80 & 0,97 \\
\hline 28) & Ahora valoro más las oportunidades que he tenido en la vida. & 4,14 & 0,90 \\
\hline 29) & Han mejorado mis habilidades sociales y comunicativas. & 4,19 & 0,84 \\
\hline 30) & $\begin{array}{l}\text { Me ha servido para aprender valores que llevaré conmigo toda la vida } \\
\text { como solidaridad, respeto, colaboración y otros. }\end{array}$ & 4,41 & 0,81 \\
\hline
\end{tabular}

Tabla 26. Utilidad en lo personal. 
(VI) Utilidad en el plano académico

Entre estos aprendizajes académicos, se destaca en primer lugar el conocimiento adquirido acerca de en qué consiste esta forma de organizar el centro en torno a las actuaciones educativas de éxito ( $M E D=4,43$; $D T=0,84)$, así como otros aspectos relacionados con la educación "que de otra manera no habrían podido aprender" $(M E D=4,40 ; D T=0,65)$, entre las que se señala el aprendizaje de metodologías de enseñanza novedosas para el estudiante (MED $=4,32$; $D T=0,89)$.

\begin{tabular}{|c|c|c|c|}
\hline & ÍTEM & MED & DT \\
\hline 31$)$ & $\begin{array}{l}\text { Participar en CdA me ha servido para aprender muchas cosas sobre } \\
\text { educación en general que de otra manera no habría podido aprender. }\end{array}$ & 4,40 & 0,65 \\
\hline 32) & $\begin{array}{l}\text { Con mi colaboración en CdA he podido ver en la práctica lo que } \\
\text { estudiamos en la facultad. }\end{array}$ & 3,52 & 1,18 \\
\hline 33) & $\begin{array}{l}\text { En CdA he aprendido en qué consiste esta forma de organizar los centros } \\
\text { y la educación denominada "Comunidades de Aprendizaje". }\end{array}$ & 4,43 & 0,84 \\
\hline 34) & $\begin{array}{l}\text { Colaborando en CdA he aprendido otras metodologías de enseñanza } \\
\text { novedosas para mí. }\end{array}$ & 4,32 & 0,89 \\
\hline 35) & Con esta experiencia ha cambiado mi idea de la enseñanza escolar. & 3,79 & 0,98 \\
\hline 36) & Mi colaboración en CdA me ha servido para despejarme de los estudios. & 3,55 & 1,24 \\
\hline
\end{tabular}

Tabla 27. Utilidad en lo académico.

(VII) Utilidad en el plano profesional

El grado de acuerdo con los ítem de este bloque referido al plano profesional también es muy alto. Así todos ellos obtienen una puntuación media entre 4,14 y 4,39 y una desviación típica entre 0,77 y 0,87, salvo en el caso del ítem 38 ("tras la colaboración en Comunidades de Aprendizaje he descubierto o redescubierto mi vocación") donde la dispersión más alta $(M E D=4,14 ; D T=I, 15)$. Se destaca que colaborando en $\mathrm{CdA}$ han podido ver el sistema educativo desde el punto de vista del docente (MED $=4,39 ; \mathrm{DT}=0,77$ ) y han podido adquirir experiencia profesional y laboral $(M E D=4,33$; $D T=0,79)$, consiguiendo competencias para el ejercicio profesional (MED $=4,27 ; \mathrm{DT}=0,27) \mathrm{y}$, en particular valorando la importancia de las altas expectativas respecto al alumnado de la educación básica (MED =4,27; $\mathrm{DT}=0,85)$. 


\begin{tabular}{|c|c|c|c|}
\hline & ÍTEM & MED & DT \\
\hline & $\begin{array}{l}\text { Participar en CdA me ha sido de ayuda en lo profesional. He adquirido } \\
\text { experiencia profesional y laboral. }\end{array}$ & 4,33 & 0,79 \\
\hline 38) & Tras la colaboración en CdA he descubierto o redescubierto mi vocación. & 4,14 & 1,15 \\
\hline 39) & $\begin{array}{l}\text { Esta colaboración me ha servido para ver la escuela desde el punto de } \\
\text { vista del profesor. }\end{array}$ & 4,39 & 0,77 \\
\hline 40) & $\begin{array}{l}\text { Me ha servido para identificarme con los escolares y tener la visión que } \\
\text { ellos poseen de la realidad }\end{array}$ & 4,14 & 0,87 \\
\hline 4I) & $\begin{array}{l}\text { Tras mi colaboración en CdA me he dado cuenta de lo importante que es } \\
\text { tener altas expectativas respecto a los escolares. }\end{array}$ & 4,27 & 0,85 \\
\hline 42) & $\begin{array}{l}\text { Mi participación en } \mathrm{CdA} \text { me ha servido para adquirir cualidades y } \\
\text { competencias necesarias para el ejercicio profesional. }\end{array}$ & 4,27 & 0,77 \\
\hline
\end{tabular}

Tabla 28. Utilidad en lo profesional

(VIII) Utilidad en el plano social

El ítem con una valoración media más alta y un mayor grado de acuerdo se encuentra en este bloque referido a cómo la participación en las CdA contribuye a adquirir conciencia de ciertas cuestiones sociales. Se trata del ítem 48 "Al colaborar en $\mathrm{CdA}$ me he dado cuenta de que todas las personas tenemos algo que enseñar $y$ todas tenemos algo que aprender" $(M E D=4,82$; $D T=0,48)$. También hay un alto grado de acuerdo en valorar la importancia de los sueños y utopías de la comunidad educativa como motor del cambio social y educativo (MED =4,49; $D T=0,69)$ y que se puede cambiar la realidad social en la que vivimos (MED =4, I8; $\mathrm{DT}=0,87)$.

Colaborar en CdA también ha servido para que los estudiantes tomen conciencia de las desigualdades sociales (MED $=4,37 ; D T=0,81$ ) y conozcan mejor la diversidad cultural creciente en nuestro entorno $(M E D=4,04 ; D T=I)$. 


\begin{tabular}{|c|c|c|c|}
\hline & ÍTEM & MED & DT \\
\hline & $\begin{array}{l}\text { Mi colaboración en CdA me ha servido para tomar conciencia de las } \\
\text { desigualdades sociales. }\end{array}$ & 4,37 & 0,81 \\
\hline 44) & $\begin{array}{l}\text { Con esta experiencia en CdA he aprendido que se puede cambiar la } \\
\text { realidad social en la que vivimos }\end{array}$ & 4,18 & 0,87 \\
\hline 45) & $\begin{array}{l}\text { Me he dado cuenta de la importancia de la comunidad educativa como } \\
\text { colectivo, de sus sueños y utopías. }\end{array}$ & 4,49 & 0,69 \\
\hline 46) & Con esta colaboración he podido conocer otras realidades sociales y económicas. & 4,32 & 0,82 \\
\hline 47) & Con esta colaboración he conocido otras pautas culturales. & 4,04 & 1,00 \\
\hline 48) & $\begin{array}{l}\text { Al colaborar en CdA me he dado cuenta de que todas las personas } \\
\text { tenemos algo que enseñar y todas tenemos algo que aprender. }\end{array}$ & 4,82 & 0,48 \\
\hline
\end{tabular}

Tabla 29. Utilidad en lo social

\section{(IX) Valoración global}

En esteúltimosub-bloqueserecogenalgunosítem que resumen lavaloración de conjunto que los estudiantes hacen de su voluntariado. Mayoritariamente afirman que sienten que tomaron una buena decisión cuando se inscribieron en esta tarea (MED=4,77; $D T=0,65)$, que ha sido una experiencia muy agradable en lo personal (MED=4,75; $D T=0,54)$, en lo profesional $(M E D=4,46 ; D T=0,87)$ y en lo académico $(M E D=4,26 ; D T=0,87)$ y que piensan recomendarla sus compañeros y compañeras (MED $=4,74 ; \mathrm{DT}=0,75)$ así como repetirla ellos mismos en futuras convocatorias (MED $=4,34$; $D T=0,75)$.

\begin{tabular}{l|c|c}
\hline \multicolumn{1}{c|}{ ÍTEM } & MED & DT \\
\hline 49) Ha sido una experiencia muy agradable en lo personal. & 4,75 & 0,54 \\
\hline 50) Ha sido una experiencia muy instructiva profesionalmente. & 4,46 & 0,76 \\
\hline 5I) He aprendido muchas cosas de utilidad para mis estudios. & 4,26 & 0,87 \\
\hline 52) Siento que tomé una buena decisión. & 4,77 & 0,65 \\
\hline 53) Si puedo, lo más probable es que repita el curso próximo. & 4,34 & 1,07 \\
\hline $\begin{array}{l}\text { 54) Pienso recomendar esta experiencia de colaboración a mis compañeros/ } \\
\text { as y amigas/os. }\end{array}$ & 4,74 & 0,75 \\
\hline
\end{tabular}

Tabla 30. Valoración global de la colaboración en CdA. 


\section{Conclusiones}

Somos conscientes de que este artículo puede resultar un poco extenso y cargado de tablas. Sin embargo, creemos que es el mejor modo, tanto en claridad como en concisión, de responder a los objetivos que nos habíamos propuesto.

En primer lugar hacer públicos los dos cuestionarios diseñados para la evaluación que hacen los estudiantes universitarios de su participación como voluntarios en las CdA. Esto nos ha llevado a mostrarlos en su totalidad y sabemos que son unos recursos bastante extensos en cuanto a número de ítem, ya que tienen I0I y 54 ítem cada uno de ellos. Es la primera vez que aparecen en una publicación de manera íntegra, lo que supone poner a disposición de la comunidad científica estos instrumentos para aquellos profesionales que están trabajando con voluntariado universitario en las distintas CdA existentes en toda España.

Pero no solo teníamos como objetivo hacer públicos los cuestionarios, sino también, dar a conocer los resultados obtenidos de los alumnos al final del curso 2013-14.

Para comenzar el comentario a este segundo objetivo señalar que las puntuaciones obtenidas en el cuestionario donde los alumnos daban su valoración acerca de las CdA (VAVU-CdA) han sido muy altas en los ítem que hacían referencia a aspectos positivos; siendo la media de los ítem referidos a la valoración general de 4,48, los ítem referentes a los aspectos positivos del trabajo con los alumno fue de 4,36 y la media de los que hacían referencia a tareas con adultos fue algo más bajo de $3,7 \mathrm{I}$. Esta puntuación más baja en la tarea con los adultos fue coherente con la puntuación media de los aspectos negativos que fue más alta $(3,45)$ que en los aspectos negativos generales $(3,07)$ y los referidos al trabajo con los niños $(2,8)$. En los aspectos negativos las desviaciones típicas también fueron más altas, lo que demuestra que la experiencia y valoración de los alumnos es diversa.

En el primer ítem de VAVU-CdA que dice "Mi valoración general de Comunidades de Aprendizaje es positiva", la puntuación de los participantes es muy alta $(4,65)$ y existe gran acuerdo entre ellos, ya que la desviación típica de este ítem es de 0,6I.

En cuanto al cuestionario de satisfacción (SAVU-CdA) en todos los bloques, que hacen referencia al grado de satisfacción en general, a cómo se han sentido con ellos mismos y con los demás, y a la utilidad de estas prácticas en el plano personal, social, profesional y académico las puntuaciones medias siempre han superado los cuatro puntos y el promedio de todas ellas ha resultado ser de 4,26 , lo que 
refleja claramente que los estudiantes se han sentido bastante satisfechos de haber participado en esta actividad de libre elección.

En cuanto a los aspectos negativos y por tanto las propuestas a mejorar que señalan los estudiantes, destacar los que puntúan por encima de 3,5 y que hacen referencia de mayor a menor a:

- Aumentar el número de voluntarios fomentando la participación de familiares y personas del barrio.

- Favorecer más la motivación de los alumnos por seguir estudiando.

- Fomentar la asistencia del alumnado a la biblioteca tutorizada.

- Incentivar más la asistencia de familiares a las tertulias.

- Comunidades de Aprendizaje exige mucho trabajoy dedicación al profesorado.

Nos parece conveniente señalar en estas conclusiones, que estos resultados van en la misma línea que el estudio realizado con la evaluación de los voluntarios en el curso anterior, y que en aquella ocasión se realizó con técnicas cualitativas de análisis de contenidos. De tal manera que podríamos hablar, de algún modo, de una triangulación en los resultados obtenidos. También, son indicador de un alto grado de coherencia entre los resultados obtenidos por ambos procedimientos, lo que repercute en la validez concurrente del instrumento.

Respecto a futuras investigaciones, y dado que se trata de un artículo en el que se describen los resultados obtenidos con toda la muestra de participantes, podrían redactarse otros artículos comparando las diferencias de puntuaciones (si las hubiese) entre subconjuntos de la muestra: varones y mujeres, según titulaciones que cursan, según la $\mathrm{CdA}$ a la que asisten, etc. Ello contribuiría a aclarar si algunas de estas variables explican la dispersión en las puntuaciones obtenidas en algunos de los bloques considerados.

Otra línea de trabajo para continuar esta investigación sería la realización de una segunda versión de los cuestionarios, replanteando cuestiones como la longitud de los mismos, especialmente del cuestionario de Valoración, la posición de algunos ítem dentro del cuestionario o la reformulación de otros. Sí nos parece un acierto continuar presentando los cuestionarios on-line para su cumplimiento dado la comodidad que supone obtener los resultados de manera instantánea sobre una hoja de cálculo. 


\section{Referencias}

Aguilera, A. y Gómez del Castillo, M. T. (20I4). Diseñando dos cuestionarios de satisfacción y valoración para el voluntariado universitario en Comunidades de Aprendizaje. En Congreso Internacional Multidisciplinar de Investigación Educativa CIMIE' I4: Investigación de calidad para mejorar la educación. Segovia: Universidad de Valladolid.

Aguilera, A., Gómez del Castillo, M. T. y Prados, Ma M. (20l4a). Cuestionario de Satisfacción de voluntariado universitario en Comunidades de Aprendizaje (SAVUCdA). Recuperado el I/I2/20I4 de <https://docs.google.com/forms/d/Iv_yTjeX8FTmeYo-I-KTko-4wkxHqPTQhvPxb_mgso/viewform?c $=0 \& w=I>$.

Aguilera, A., Gómez del Castillo, M. T. y Prados, Ma M. (20l4b). Cuestionario de Valoración de Comunidades de Aprendizaje por el Voluntariado Universitario (VAVUCdA). Recuperado el I/I2/20I4 de <https://docs.google.com/forms/d/I 6ajLFRTqvR flLesGhbgbkdlu9cGrTsET7hccgF4Nio/viewform?c=0\&w $=\mid>$.

Aguilera, A., Ignacio, M. J., Prados, M. M., Sánchez, V., Gómez del Castillo, M. T., Ordóñez, R. y Rodríguez, M. (20I4). La participación de alumnos en comunidades de aprendizaje. En IV Jornadas Innovación Docente: Abriendo caminos para la mejora educativa. Sevilla: Universidad de Sevilla.

Aretxaga, L. y Landaluce, L. M. (2005). La interculturalidad en el IES Mungia BHI. En Congreso para el diálogo intercultural e interreligioso (pp.2I4-220). Bilbao.

Biesta, G. (20I2).The future of teacher education: Evidence, competence or wisdom? Research on Steiner Education, 3 (I), 8-2I.

Elboj, C., Puigdellívol, I., Soler, M., y Valls, R. (2002). Comunidades de aprendizaje. Transformar la educación. Barcelona: Graó.

Flecha, R., Racionero, S., Tintoré, M. y Arbós, A. (20I4). Actuaciones de éxito en la universidad. Hacia la excelencia tomando las mejores universidades como modelo. Multidisciplinary Journal of Educational Research, 4 (2), I3I-I 50.

Freire, P. (2005). Cartas a quien pretende enseñar. México: Siglo XXI.

Gómez del Castillo, M. T., Aguilera, A. y Prados, M. M. (20I5). El voluntariado de la Universidad de Sevilla en los grupos interactivos. Revista Profesorado (en prensa).

Imbernón, F. (20I2). La formación del profesorado universitario: orientaciones y desorientaciones. Las prácticas de formación del profesorado universitario. En J. 
B. Martínez (Coord.), Innovación en la universidad: prácticas, políticas y retóricas (pp. 85-103). Barcelona: Graó.

INCLUD-ED (20II). Actuaciones de éxito en las escuelas europeas. Madrid: Ministerio de Educación.

Red Utopía y Educación (2012). Bibliotecas y aulas de informática tutorizadas. Recuperado el 12/2/2015 de <http://www.utopiayeducacion.com/2006/06/ bibliotecas-y-aulas-de-infomtica.html $>$.

Rodríguez de Guzmán, J. (20I2). Comunidades de aprendizaje y formación del profesorado. Tendencias Pedagógicas, 19, 67-86.

Rubia, B., Jorri, I. y Anguita, R. (2009). Aprendizaje colaborativo y TIC. En J. De Pablos (Coord.), Tecnología Educativa. La formación del profesorado en la era digital. Málaga: Aljibe.

Valls, R. y Padros, M. M. (20I I). Using dialogic research to overcome poverty: From principles to action. European Journal of Education, 46 (2), I73-183. 